resembles the phenotype of the baz group mutants in many respects, but becomes manifest slightly later ${ }^{1,3,6}$, suggesting that $b a z$ may function upstream of $c r b$. The proteins encoded by the baz and $c r b$ group genes are all localized at the apical plasma membrane and are enriched in the subapical region (SAR) adjacent to the $\mathrm{ZA}^{4-8}$ (Fig. 2). The SAR of Drosophila epithelia is defined as the portion of the plasma membrane that borders apically to the ZA. Interestingly, the SAR corresponds in position, protein composition, and even in some functional aspects, to the tight junction in vertebrates, although it differs from the tight junction in its ultrastructural appearance ${ }^{6}$. Bilder et al. ${ }^{1}$ now show that in the absence of Arm, apical localization of both Baz and Crb is never established. Crb is lost from the apical membrane in baz mutant embryos, whereas Baz is localized normally in $\mathrm{crb}$ mutant embryos at the same developmental stage, supporting the view that $\mathrm{Baz}$ functions upstream of $\mathrm{Crb}$ and is required for its proper localization ${ }^{1}$.

Mutants of the $d l g$ group also show a loss of the ZA, abnormal cell shapes and multilayering of the epidermis, but their phenotypes display some important differences to those of the baz and crb groups: whereas in mutants of the latter two groups, the apical plasma membrane domain appears to be strongly reduced or absent, it is enlarged in mutants of the $d l g$ group ${ }^{1,2,9,10}$. In contrast to $b a z$ and $c r b$ mutants, there is no ectopic cell death in $\mathrm{dlg}$ mutants. At gastrulation, Scrib and Dlg are enriched basally to the newly emerging $\mathrm{ZA}$, whereas $\mathrm{Lgl}$ is spread more evenly along the basolateral plasma membrane and is also present in the cytoplasm $^{9,10}$ (Fig. 2). In common with the members of the baz and crb groups, the proteins of the $d l g$ group are mutually dependent on each other for their correct subcellular localization ${ }^{4,5,7-9}$.

These observations raise the obvious question of how these three groups of genes interact with each other to control cell polarity and ZA assembly. In a genetically accessible model organism such as Drosophila, this question is most easily addressed by the analysis of double-mutant phenotypes. The underlying rationale is as follows: if the phenotype of two single mutants is similar and the double mutant shows the same phenotype as any of the single mutants, the two genes probably function in a single linear pathway. However, if the phenotype of the double mutant is different from any of the single mutant phenotypes, then the two genes may function redundantly or in parallel pathways. Finally, if the phenotypes of the single mutants are distinct, but the phenotype of the double mutant is identical to one of the single mutants, then the resulting predominant phenotype is epistatic. The latter result allows us to conclude that the epistatic gene, whose mutant phenotype predominates, functions at a higher hierarchical position in the order of a common process. To get a better feeling for what epistasis means, think of the following example: if you are homozygous for a mutation that causes baldness, it does not matter whether you carry alleles for blond or black hair colour on another locus, as the bald phenotype predominates and is epistatic to the hair colour phenotype.

\title{
ZAP-ping T-cell activation
}

Antigen stimulation of any $\mathrm{T}$ cell initiates a complex series of events, culminating in $\mathrm{T}$ cell activation and an immune response. At the heart of this response is a cascade of signalling pathways, primarily — but not exclusively — involving mitogenactivated protein kinases (MAPKs). ZAP-70, a Syk-related tyrosine kinase, is also essential for an immune response in activated $\mathrm{T}$ cells, and although this kinase regulates MAPK activation, its precise targets have remained elusive. In the latest issue of Nature Immunology (Nature Immunol. 4, 46-50 (2003)) Alonso et al. identify at last a potential target of ZAP-70 in the regulation of T-cell activation.

Common sense dictates that the activation of any immune response must be highly regulated in both intensity and duration. Regulating the activity of kinase signalling pathways, such as those initiated by MAPK, through protein phosphatases (PPases) is crucial after any $\mathrm{T}$ cell-antigen interaction. These PPases curtail the kinase response in a temporal manner, depending on the type of $\mathrm{T}$ cell activated or the antigen presented to the cells. VHR (Vaccinia virus VH-1-related dual-specific protein phosphatase) is one such PPase that has an essential role in regulating T-cell activation through inhibition of the extracellular signal-regulated kinases (Erk/MAPK) or Jun kinase (JNK) signal cascades. In this context, it was unclear whether ZAP-70 is involved in VHR-mediated regulation.

Through expression studies in P116 cells lacking functional ZAP-70, Alonso et al. indicate that VHR activity is probably regulated by ZAP-70-mediated phosphorylation of VHR-Tyr 138. Rescue experiments in cells co-expressing either wild-type VHR or $\mathrm{VHR}^{\mathrm{Y} 138 \mathrm{~F}}$ (in which the putative tyrosine phosphorylation site is mutated to phenylalanine) with recombinant ZAP-70 indicate that phosphorylation of this particular residue by ZAP-70 is essential for activation of VHR. Phosphorylation of VHR-Tyr 138 is also critical for inhibition of the Erk pathway in activated $\mathrm{T}$ cells. The localization of phosphorylated VHR to the immune

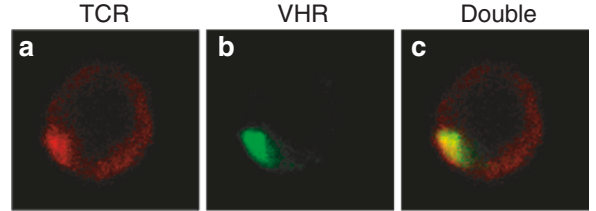

Figure 1 CD8-positive T cells incubated with antigen-presenting cells. Confocal microscope images showing localization of T-cell receptor (a) localization of VHR (b) and colocalization of TCR Tcell receptor and VHR to sites of the immune synapse (c).

synapse after antigen presentation to cells (see Figure) provides further correlative evidence to suggest that phosphorylated VHR is important in regulating T-cell activation.

Therefore, VHR-mediated inhibition of the Erk pathway after T-cell activation may involve phosphorylation of ZAP-70, making it one of the few known targets of this enzyme. This would ensure that while both Erk and ZAP-70 would be activated after T cell activation, ZAP-70 would then curtail the Erk response through phosphorylation and activation of the VHR PPase. However, as the $\mathrm{VHR}^{\mathrm{Y} 138 \mathrm{~F}}$ mutant potentiates Erk signalling, the role of VHR in augmenting T-cell activation is not straightforward. These results raise the interesting possibilities that phosphorylation of VHR might not affect the catalytic activity of the PPase, but may cause alterations in protein localization, protein conformation or substrate specificity, all of which could be equally important in controlling the temporal regulation of MAPKs in activated T cells.

SARAH GREAVES 